\title{
Assessment of Soil for Increasing the Yield of Mustard in Northern Hills, Chhattisgarh, India
}

\author{
Alakh Gour $^{1 *}$, Dogendra Kumar Sahu ${ }^{2}$ and Deepak Thakur ${ }^{1}$ \\ ${ }^{1}$ Krishi Vigyan Kendra, Balod, ${ }^{2}$ Krishi Vigyan Kendra, Jashpur, Indira Gandhi Krishi \\ Vishwavidyalaya, Raipur, 492001, Chhattisgarh, India
}

*Corresponding author

\begin{tabular}{l} 
Ke y w o r d s \\
Pathalgaon and \\
Bagicha Block, \\
Chemical \\
properties, Mustard \\
yield, \\
No. of Siliqua \\
\hline $\begin{array}{l}\text { Article Info } \\
\text { Accepted: } \\
22 \text { October } 2019 \\
\text { Available Online: } \\
\text { 10 November } 2019\end{array}$ \\
\hline
\end{tabular}

A B S T R A C T
A survey of chemical properties of soil of Pathalgaon and Bagicha Block of Jashpur District, Chhattisgarh India was carried out in year 2017-18. 27 Soil samples were collected at a depth of 0-15 from 3 Village- Kamarema, Gala, Kodkelkhajri. Soil sample were analyzed for $\mathrm{pH}, \mathrm{EC}, \mathrm{OC}$ and N, P, $\mathrm{K}, \mathrm{Zn}$ and $\mathrm{S}$. The value of $\mathrm{pH}, \mathrm{EC}, \mathrm{OC}$ is found in ranges from 5.42 to 6.51 , $0.04-0.16 \mathrm{dSm}^{-1}, 0.44$ to 0.63 per cent respectively. and $\mathrm{N}, \mathrm{P}, \mathrm{K}, \mathrm{S}, \mathrm{Zn}$ is found in ranges from 182.2 to $236.25 \mathrm{~kg} \mathrm{ha}^{-1}, 9.40$ to $16.6 \mathrm{~kg} \mathrm{ha}^{-1}, 101.4$ to $170.3 \mathrm{~kg} \mathrm{ha}^{-1}, 25.87$ to $33.2 \mathrm{~kg} \mathrm{ha}^{-1}, 1.70$ to $4.74 \mathrm{~kg} \mathrm{ha}^{-1}$ respectively. The $\mathrm{pH}$ is acidic to neutral, Electrical Conductivity is Low to high. The status of organic carbon percent and nitrogen is low to medium, the status of phosphorus, potassium is Low to medium and sulphur is medium in soil, and $\mathrm{Zn}$ is high and Maximum yield $13.40 \mathrm{q} \mathrm{ha}^{-1}$ and minimum $8.20 \mathrm{q} \mathrm{ha}^{-1}$. The maximum siliqua are often 110.02 an minimum no. of siliqua 70.09 based on soil test and their recommendation.

\section{Introduction}

Mustard occupies a prestigious place in Indian agriculture due to their vital role in the sustainable economy of the country. Vegetable oil (edible) plays a significant role in human nutrition. Indian mustard is one of the most important winter oil seed crops. Sulphur improves the quality of mustard by increasing the oil contents, protein content and several fatty acids. Sulphur helps in chlorophyll formation and also encourages vegetative growth. It also helps in the reductionoxidation reactions in the respiration. The importance of micronutrients application in increasing crop production has been recognized in India (Dubey et al., 2013). Soil is the basic resource for agriculture and its 
proper management is essential to sustain agricultural production and maintain soil productivity. Soil testing is one of the best available tools, to ascertain the physical characteristics \& nutrient status of a field. Soil test-based fertility management is an effective tool for increasing productivity of agricultural soils that have high degree of spatial variability resulting from the combined effects of physical, chemical or biological processes (Goovaerts, 1998).

The state receives annual rainfall ranging from less than $1200 \mathrm{~mm}$ to greater than $1600 \mathrm{~mm}$ in different areas. There are a number of types of soil found in Chhattisgarh area but there are four major types namely Kanhar, Matasi, Dorsa and Bhata.

The soils of the region are deficient in important mineral nutrients like nitrogen, phosphorous, lime and potash, which are concentrated in the lower parts of the soil layer (Tripathi and Bhardwaj, 2016). At present 27 districts are there in Chhattisgarh. Chhattisgarh is situated between 17-23.70 N latitude and 80.40-83.380 E longitude in Central eastern part of India. The total geographical area of the state is 136.03 thousand sq. km.

Geographically, Chhattisgarh is divided into three distinct land areas viz.

Chhattisgarh Plains

Bastar Plateau and

Northern Hill Zones

India is amongst the largest vegetable oil economic in the world. Mustard is rich in minerals like calcium, manganese, copper, iron, selenium, zinc, vitamin $\mathrm{A}, \mathrm{B}, \mathrm{C}$ and proteins. $100 \mathrm{~g}$ mustard seed contains $508 \mathrm{kcal}$ energy, 28.09 g carbohydrates, 26.08 g proteins, $36.24 \mathrm{~g}$ total fat and $12.2 \mathrm{~g}$ dietary fibre. Sulphur plays a significant role in increasing production especially in oilseeds (Upadhyay et al., 2016).

\section{Materials and Methods}

Pathalgaon and Bagicha is a Block located in Jashpur district Placed in rural region of Chhattisgarh, it is one among the blocks of Jashpur District. Pathlgaon is located at $22^{\circ} 30^{\prime} 378^{\prime \prime} \mathrm{N}$ latitude $83^{\circ} 29^{\prime} 154^{\prime \prime} \mathrm{E}$ longitute) normal rainfall is $1450.0 \mathrm{~mm}$ (20172018) and average annual rainfall $1726.0 \mathrm{~mm}$. The region generally experiences hot, sub humid climate. It has an average elevation of $348 \mathrm{~m}(1,142 \mathrm{ft})$.

\section{Sampling and analysis}

The 27 soil samples were collected with0-15 $\mathrm{cm}$. depths from 3 Village- Kamarema, Gala, Kodkelkhajri.

The $\mathrm{pH}, \mathrm{Ec}, \mathrm{OC}, \mathrm{N}, \mathrm{P}, \mathrm{K}, \mathrm{S}, \mathrm{Zn}$ was determined by Mrida pariksahak Equipment, Developed by ICAR-Indian Institute of Soil Science, Bhopal.

\section{Results and Discussion}

\section{Soil pH}

The Lowest value of $\mathrm{pH}$ found in Kamarema at depth $(0-15 \mathrm{~cm}) 5.42$ and highest value in Kodkelkhajri at depth $(0-15 \mathrm{~cm}) 6.51$ and $\mathrm{pH}$ was found to be significant at different villages (Fig. 1, Table 1 and 2).

\section{Electrical conductivity (EC)}

The lowest value of electrical conductivity found in Kamarema at depth $(0-15 \mathrm{~cm}) 0.04$ $\mathrm{dSm}^{-1}$ and highest value in Kodkelkhajri at depth $(0-15 \mathrm{~cm}) 0.16 \mathrm{dSm}^{-1}$ (Fig. 2). The EC was found to be significant at different villages. 


\section{Organic carbon (OC) percent}

The lowest value of organic carbon percent is found in soil of Kamarema at depth $(0-15 \mathrm{~cm})$ 0.44 per cent and highest value in soil of Kodkelkhajri at depth $(0-15 \mathrm{~cm}) 0.63$ per cent.

The OC (\%) was found to be significant at different villages (Fig. 3).

\section{Available nitrogen $\left(\mathrm{kg} \mathrm{ha}^{-1}\right)$}

The Lowest value of nitrogen is found in soil of Kamarema at depth $(0-15 \mathrm{~cm}) 182.2 \mathrm{~kg} \mathrm{ha}^{-1}$ and highest value in soil of Kodkelkhajriat depth $(0-15 \mathrm{~cm}) 236.25 \mathrm{~kg} \mathrm{ha}^{-1}$. The available nitrogen $\left(\mathrm{kg} \mathrm{ha}^{-1}\right)$ was found to be significant at different villages (Fig. 4).

\section{Available phosphorus ( $\left.\mathrm{kg} \mathrm{ha}^{-1}\right)$}

The value of available phosphorus $\left(\mathrm{kg} \mathrm{ha}^{-1}\right)$ is found in varied from 10.4 to $20.2 \mathrm{~kg} \mathrm{ha}^{-1}$. The lowest value of phosphorus is found in soil of
Kodkelkhajriat depth $(0-15 \mathrm{~cm}) 9.40 \mathrm{~kg} \mathrm{ha}^{-1}$ and highest value in soil of Kamarema at depth $(0-15 \mathrm{~cm}) 16.6 \mathrm{~kg} \mathrm{ha}^{-1}$. The available phosphorus $\left(\mathrm{kg} \mathrm{ha}{ }^{-1}\right)$ was found to be significant at different villages (Fig. 5).

\section{Available potassium $\left(\mathrm{kg} \mathrm{ha}^{-1}\right)$}

The lowest value of potassium is found in soil of Kodkelkhajri at depth $(0-15 \mathrm{~cm}) 101.4 \mathrm{~kg}$ $\mathrm{ha}^{-1}$ and highest value in soil of Kamarema at depth $(0-15 \mathrm{~cm}) 170.3 \mathrm{~kg} \mathrm{ha}^{-1}$. The available potassium $\left(\mathrm{kg} \mathrm{ha}{ }^{-1}\right)$ was found to be significant at different villages (Fig. 6).

\section{Available sulphur (kg ha-1)}

The lowest value of sulphur is found in soil of Kodkelkhajri at depth $(0-15 \mathrm{~cm}) 25.87 \mathrm{~kg} \mathrm{ha}^{-1}$ and highest value in soil of Kamarema at depth $(0-15 \mathrm{~cm}) 33.2 \mathrm{~kg} \mathrm{ha}^{-1}$. The available sulphur $\left(\mathrm{kg} \mathrm{ha}^{-1}\right)$ was found to be significant at different depths and villages (Fig. 7).

Table.1 Rating chart for soil test values and their nutrient indices based on the specific rating chart modified from Brajendra et al., (2014)

\begin{tabular}{|c|c|c|c|c|}
\hline Soil property & Unit & \multicolumn{3}{|c|}{ Range } \\
\hline Soil $\mathrm{pH}$ & $\mathrm{pH}$ unit & $<6.5$ (Acidic) & $6.5-7.5$ (Neutral) & $\begin{array}{c}>8.0 \\
\text { (Alkaline) }\end{array}$ \\
\hline $\begin{array}{c}\text { Electrical } \\
\text { conductivity }\end{array}$ & $\mathrm{dSm}^{-1}$ & $<1.0$ (Normal) & $1.0-2.0$ (Critical) & $\begin{array}{c}>2.0 \\
\text { (Injurious) }\end{array}$ \\
\hline $\begin{array}{c}\text { Organic carbon } \\
\text { Available nitrogen } \\
(\mathrm{N})\end{array}$ & $\mathrm{Percent}^{-1}$ & $<0.5$ (Low) & $0.5-0.75$ (Medium) & $>0.75$ (High) \\
\hline $\begin{array}{c}\text { Available } \\
\text { phosphorus }\left(\mathrm{P}_{2} \mathrm{O}_{5}\right)\end{array}$ & $\mathrm{kg} \mathrm{ha}^{-1}$ & $<280$ (Low) & $280-560$ (Medium) & $>560$ (High) \\
\hline $\begin{array}{c}\text { Available } \\
\text { potassium }\left(\mathrm{K}_{2} \mathrm{O}\right)\end{array}$ & $\mathrm{kg} \mathrm{ha}^{-1}$ & $<135$ (Low) & $135-335$ (Medium) & $>335$ (High) \\
\hline $\begin{array}{c}\text { Available sulphur } \\
(\mathrm{S})\end{array}$ & $\mathrm{kg} \mathrm{ha}^{-1}$ & $<22.5$ (Low) & $22.5-35$ (Medium) & $>35$ (High) \\
\hline $\begin{array}{c}\text { Available zinc } \\
(\mathrm{Zn})\end{array}$ & $\mathrm{ppm}^{-12.5-25 \text { (Medium) }}$ & $>25$ (High) & $>1.0$ (High) \\
\hline
\end{tabular}




\section{Int.J.Curr.Microbiol.App.Sci (2019) 8(11): 2331-2341}

Table.2 Analysis results of chemical parameters of soil samples of Pathalgaon and Bagicha Block in Jashpur District, Chhattishgarh

\begin{tabular}{|c|c|c|c|c|c|c|c|c|c|c|c|c|c|c|c|c|c|c|c|c|c|c|c|c|}
\hline \multirow{3}{*}{ 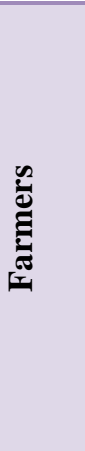 } & \multicolumn{3}{|c|}{$\begin{array}{l}\mathbf{p H} \\
(\mathbf{w} / \mathbf{v})\end{array}$} & \multicolumn{3}{|c|}{$\underset{\left(\mathbf{d S m}^{-1}\right)}{\mathbf{E C}}$} & \multicolumn{3}{|c|}{$\begin{array}{c}\text { Organic } \\
\text { Carbon }(\%)\end{array}$} & \multicolumn{3}{|c|}{$\begin{array}{l}\text { Nitrogen } \\
\left(\mathrm{Kg} \mathrm{ha}^{-1}\right)\end{array}$} & \multicolumn{3}{|c|}{$\begin{array}{l}\text { Phosphorous } \\
\left(\mathrm{Kg} \mathrm{ha}^{-1}\right)\end{array}$} & \multicolumn{3}{|c|}{$\begin{array}{l}\text { Potassium } \\
\left(\mathrm{Kg} \mathrm{ha}^{-1}\right)\end{array}$} & \multicolumn{3}{|c|}{$\begin{array}{l}\text { Sulphur } \\
\left(\mathrm{Kg} \mathrm{ha}^{-1}\right)\end{array}$} & \multicolumn{3}{|c|}{$\begin{array}{c}\text { Zinc } \\
\left(\mathbf{K g ~ h a}^{-1}\right)\end{array}$} \\
\hline & \multicolumn{3}{|c|}{ Village Name } & \multicolumn{3}{|c|}{ Village Name } & \multicolumn{3}{|c|}{ Village Name } & \multicolumn{3}{|c|}{ Village Name } & \multicolumn{3}{|c|}{ Village Name } & \multicolumn{3}{|c|}{ Village Name } & \multicolumn{3}{|c|}{ Village Name } & \multicolumn{3}{|c|}{ Village Name } \\
\hline & 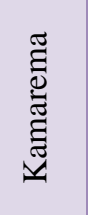 & సँّ & 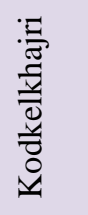 & 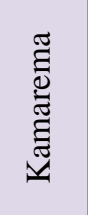 & $\frac{\pi}{\tilde{J}}$ & 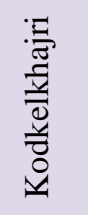 & 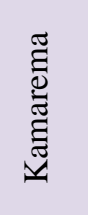 & $\frac{\frac{\pi}{\pi}}{0}$ & 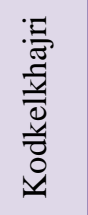 & 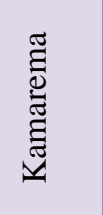 & $\frac{\pi}{\tilde{J}}$ & 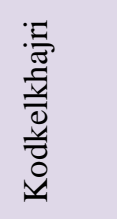 & 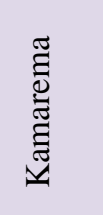 & $\frac{\pi}{\tilde{J}}$ & 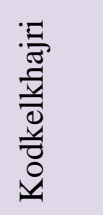 & 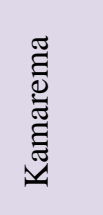 & $\frac{\pi}{\tilde{J}}$ & 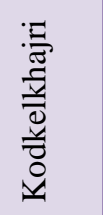 & 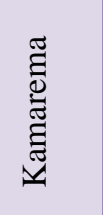 & $\frac{\pi}{\tilde{J}}$ & 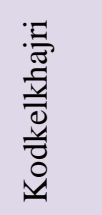 & 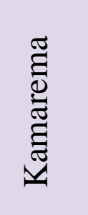 & $\frac{\pi}{\tilde{J}}$ & 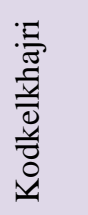 \\
\hline $\mathbf{F}_{1}$ & 5.94 & 5.54 & 5.54 & 0.06 & 0.11 & 0.11 & 0.46 & 0.47 & 0.44 & 182.2 & 209.0 & 212.50 & 11.60 & 12.60 & 11.60 & 150.0 & 126.5 & 123.5 & 26.71 & 33.20 & 32.60 & 1.94 & 2.10 & 4.74 \\
\hline $\mathbf{F}_{2}$ & 6.10 & 6.19 & 6.19 & 0.09 & 0.08 & 0.08 & 0.44 & 0.51 & 0.47 & 190.1 & 216.0 & 214.60 & 16.60 & 12.40 & 14.50 & 154.6 & 121.9 & 109.3 & 29.31 & 32.60 & 31.26 & 1.84 & 3.12 & 3.21 \\
\hline $\mathbf{F}_{3}$ & 5.64 & 6.20 & 6.20 & 0.11 & 0.12 & 0.09 & 0.47 & 0.60 & 0.44 & 184.2 & 211.3 & 219.60 & 14.20 & 13.90 & 11.60 & 140.3 & 131.9 & 111.6 & 33.20 & 31.26 & 30.10 & 1.96 & 2.91 & 3.96 \\
\hline $\mathbf{F}_{4}$ & 5.54 & 6.10 & 5.96 & 0.08 & 0.06 & 0.11 & 0.51 & 0.44 & 0.47 & 187.5 & 213.0 & 213.90 & 11.20 & 16.10 & 14.70 & 133.2 & 121.3 & 120.6 & 32.60 & 26.71 & 29.31 & 1.77 & 2.46 & 3.46 \\
\hline$F_{5}$ & 6.19 & 5.94 & 5.64 & 0.12 & 0.09 & 0.08 & 0.60 & 0.47 & 0.51 & 201.2 & 221.2 & 217.90 & 13.60 & 15.40 & 10.50 & 144.7 & 114.9 & 108.6 & 31.26 & 29.31 & 33.20 & 2.10 & 3.61 & 2.91 \\
\hline$F_{6}$ & 6.20 & 6.10 & 5.54 & 0.08 & 0.11 & 0.12 & 0.42 & 0.60 & 0.63 & 191.3 & 219.0 & 224.60 & 15.40 & 11.80 & 11.90 & 161.8 & 123.6 & 103.6 & 30.10 & 33.20 & 32.60 & 1.99 & 3.21 & 3.01 \\
\hline $\mathbf{F}_{7}$ & 5.92 & 5.64 & 6.19 & 0.11 & 0.08 & 0.08 & 0.48 & 0.42 & 0.51 & 186.9 & 213.1 & 223.60 & 12.40 & 10.60 & 15.90 & 146.5 & 128.9 & 118.6 & 29.36 & 32.60 & 26.71 & 2.30 & 1.96 & 3.67 \\
\hline $\mathbf{F}_{8}$ & 5.78 & 5.54 & 6.51 & 0.04 & 0.12 & 0.14 & 0.47 & 0.47 & 0.60 & 195.6 & 217.0 & 231.50 & 11.90 & 14.60 & 11.80 & 133.3 & 120.9 & 146.8 & 32.32 & 31.26 & 29.31 & 1.70 & 3.21 & 2.98 \\
\hline $\mathbf{F}_{9}$ & 5.42 & 6.10 & 6.31 & 0.09 & 0.11 & 0.16 & 0.53 & 0.51 & 0.42 & 212.0 & 214.0 & 236.25 & 12.60 & 11.90 & 9.40 & 120.5 & 124.5 & 101.4 & 28.27 & 30.10 & 25.87 & 3.37 & 2.89 & 3.12 \\
\hline
\end{tabular}


Table.3 Recommendation of chemical fertilizer Based on Soil test of Pathalgaon and Bagicha Block in Jashpur District, Chhattishgarh

\begin{tabular}{|c|c|c|c|c|c|c|c|c|c|c|c|c|c|c|c|}
\hline \multirow{2}{*}{$\begin{array}{c}\text { Farme } \\
\text { rs }\end{array}$} & \multicolumn{5}{|c|}{ Kamarema } & \multicolumn{5}{|c|}{ Gala } & \multicolumn{5}{|c|}{ Kodkelkhajri } \\
\hline & $\begin{array}{c}\mathrm{N} \\
\text { applied } \\
\text { (kg/ha) }\end{array}$ & $\begin{array}{c}\mathbf{P} \\
\text { applied } \\
\text { (kg/ha) }\end{array}$ & $\begin{array}{c}\text { K } \\
\text { applied } \\
\text { (kg/ha) }\end{array}$ & $\begin{array}{c}\text { S } \\
\text { applied } \\
\text { (kg/ha) }\end{array}$ & $\begin{array}{c}\text { Zn } \\
\text { applied } \\
\text { (kg/ha) }\end{array}$ & $\begin{array}{c}\mathrm{N} \\
\text { applied } \\
\text { (kg/ha) }\end{array}$ & $\begin{array}{c}\text { P } \\
\text { applied } \\
\text { (kg/ha) }\end{array}$ & $\begin{array}{c}\text { K } \\
\text { applied } \\
(\mathrm{kg} / \mathrm{ha})\end{array}$ & $\begin{array}{c}\text { S } \\
\text { applie } \\
\text { d } \\
\text { (kg/ha) }\end{array}$ & $\begin{array}{c}\text { Zn } \\
\text { applie } \\
\text { d } \\
\text { (kg/ha) }\end{array}$ & $\begin{array}{c}\mathrm{N} \\
\text { applie } \\
\mathrm{d}(\mathrm{kg} / \mathrm{h} \\
\text { a) }\end{array}$ & $\begin{array}{c}P \\
\text { applie } \\
\text { d(kg/h } \\
\text { a) }\end{array}$ & $\begin{array}{c}\mathrm{K} \\
\text { applie } \\
\text { d(kg/h } \\
\text { a) }\end{array}$ & $\begin{array}{c}\text { s } \\
\text { applie } \\
\text { d(kg/h } \\
\text { a) }\end{array}$ & $\begin{array}{c}\text { Zn } \\
\text { applie } \\
\text { d(kg/h } \\
\text { a) }\end{array}$ \\
\hline $\mathbf{F}_{1}$ & 106.00 & 60.00 & 30.00 & 0.00 & 0.00 & 106.00 & 60.00 & 40.00 & 0.00 & 0.00 & 106.00 & 60.00 & 40.00 & 0.00 & 0.00 \\
\hline $\mathbf{F}_{2}$ & 106.00 & 60.00 & 30.00 & 0.00 & 0.00 & 106.00 & 60.00 & 40.00 & 0.00 & 0.00 & 106.00 & 60.00 & 40.00 & 0.00 & 0.00 \\
\hline $\mathbf{F}_{3}$ & 106.00 & 60.00 & 30.00 & 0.00 & 0.00 & 106.00 & 60.00 & 40.00 & 0.00 & 0.00 & 106.00 & 60.00 & 40.00 & 0.00 & 0.00 \\
\hline $\mathbf{F}_{4}$ & 106.00 & 60.00 & 30.00 & 0.00 & 0.00 & 106.00 & 60.00 & 40.00 & 0.00 & 0.00 & 106.00 & 60.00 & 40.00 & 0.00 & 0.00 \\
\hline $\mathbf{F}_{5}$ & 106.00 & 60.00 & 30.00 & 0.00 & 0.00 & 106.00 & 60.00 & 40.00 & 0.00 & 0.00 & 106.00 & 60.00 & 40.00 & 0.00 & 0.00 \\
\hline $\mathbf{F}_{6}$ & 106.00 & 60.00 & 30.00 & 0.00 & 0.00 & 106.00 & 60.00 & 40.00 & 0.00 & 0.00 & 106.00 & 80.00 & 40.00 & 0.00 & 0.00 \\
\hline $\mathbf{F}_{7}$ & 106.00 & 60.00 & 30.00 & 0.00 & 0.00 & 106.00 & 60.00 & 40.00 & 0.00 & 0.00 & 106.00 & 80.00 & 40.00 & 0.00 & 0.00 \\
\hline $\mathbf{F}_{8}$ & 106.00 & 60.00 & 30.00 & 0.00 & 0.00 & 106.00 & 60.00 & 40.00 & 0.00 & 0.00 & 106.00 & 80.00 & 40.00 & 0.00 & 0.00 \\
\hline $\mathbf{F}_{9}$ & 106.00 & 60.00 & 30.00 & 0.00 & 0.00 & 106.00 & 60.00 & 40.00 & 0.00 & 0.00 & 106.00 & 80.00 & 40.00 & 0.00 & 0.00 \\
\hline
\end{tabular}


Table.4 Siliqua per plant

\begin{tabular}{|c|c|c|c|}
\hline Treatment & Kamarima & Gala & Kudkelkhajari \\
\hline Farmer -1 & 85.57 & 96.98 & 85.57 \\
\hline Farmer -2 & 85.57 & 72.94 & 89.65 \\
\hline Farmer -3 & 92.91 & 98.61 & 97.8 \\
\hline Farmer- 4 & 96.98 & 93.72 & 96.17 \\
\hline Farmer -5 & 66.83 & 101.87 & 109.21 \\
\hline Farmer -6 & 70.90 & 72.94 & 81.5 \\
\hline Farmer -7 & 70.09 & 110.02 & 77.42 \\
\hline Farmer -8 & 81.5 & 101.87 & 79.05 \\
\hline Farmer -9 & 80.02 & 89.65 & 85.57 \\
\hline
\end{tabular}

Table.5 Mustard yield Q/ ha

\begin{tabular}{|c|c|c|c|}
\hline Treatment & Kamarima Village & Gala Village & Kudkelkhajari Village \\
\hline Farmer -1 & 10.50 & 11.90 & 10.50 \\
\hline Farmer -2 & 10.50 & 8.95 & 11.00 \\
\hline Farmer -3 & 11.40 & 12.10 & 12.00 \\
\hline Farmer- 4 & 11.90 & 11.50 & 11.80 \\
\hline Farmer -5 & 8.20 & 12.50 & 13.40 \\
\hline Farmer -6 & 8.70 & 8.95 & 10.00 \\
\hline Farmer -7 & 8.60 & 13.50 & 9.50 \\
\hline Farmer -8 & 10.00 & 12.50 & 9.70 \\
\hline Farmer -9 & 10.80 & 11.00 & 10.50 \\
\hline
\end{tabular}

Fig.1 Availability of $\mathrm{pH}$ at different village of Jashpur District

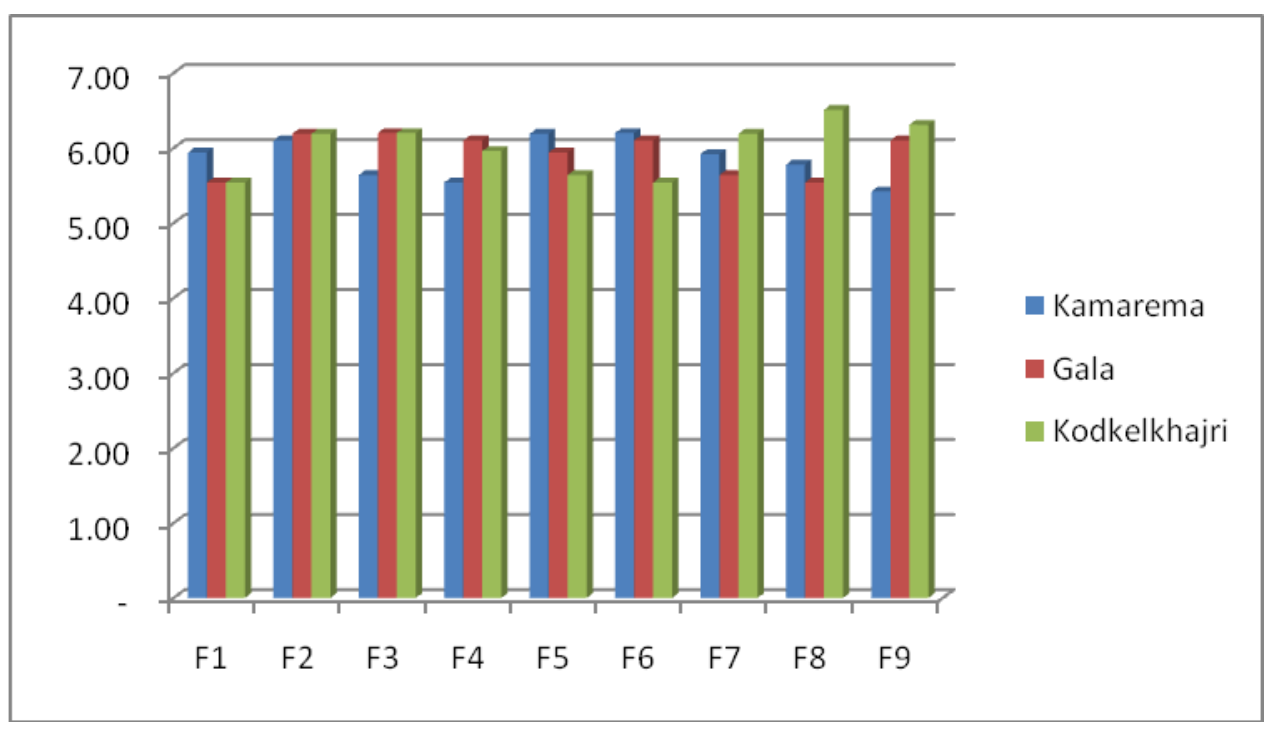


Fig.2 Availability of Ec at different village of Jashpur District

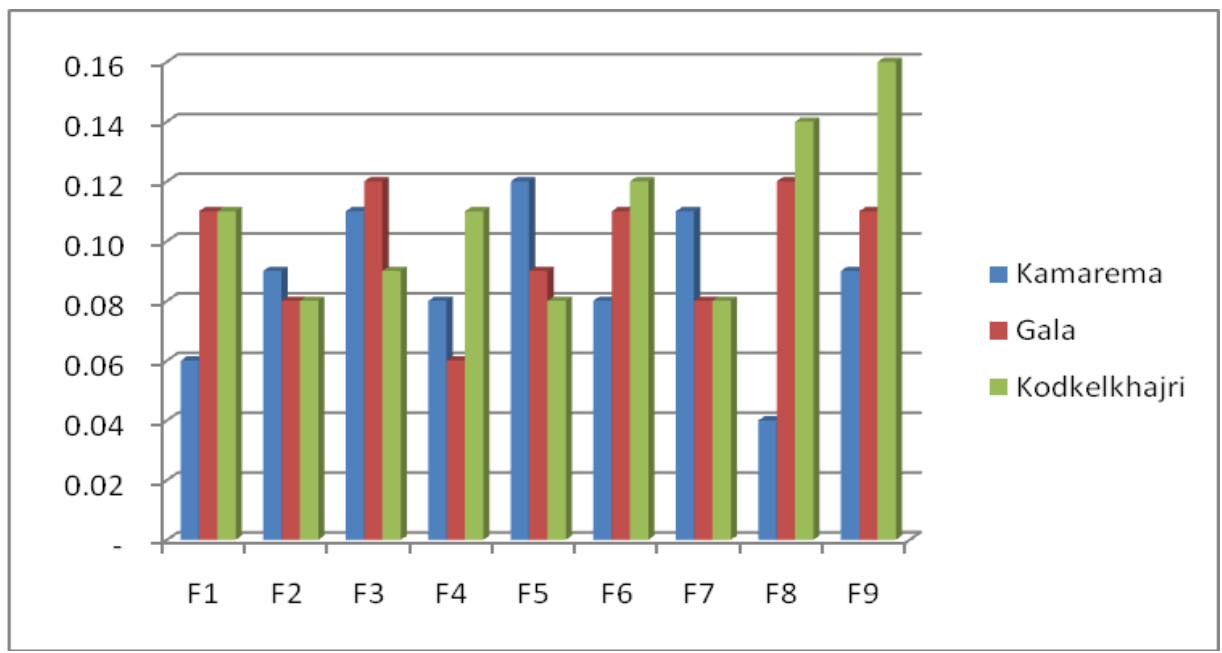

Fig.3 Availability of OC at different village of Jashpur District

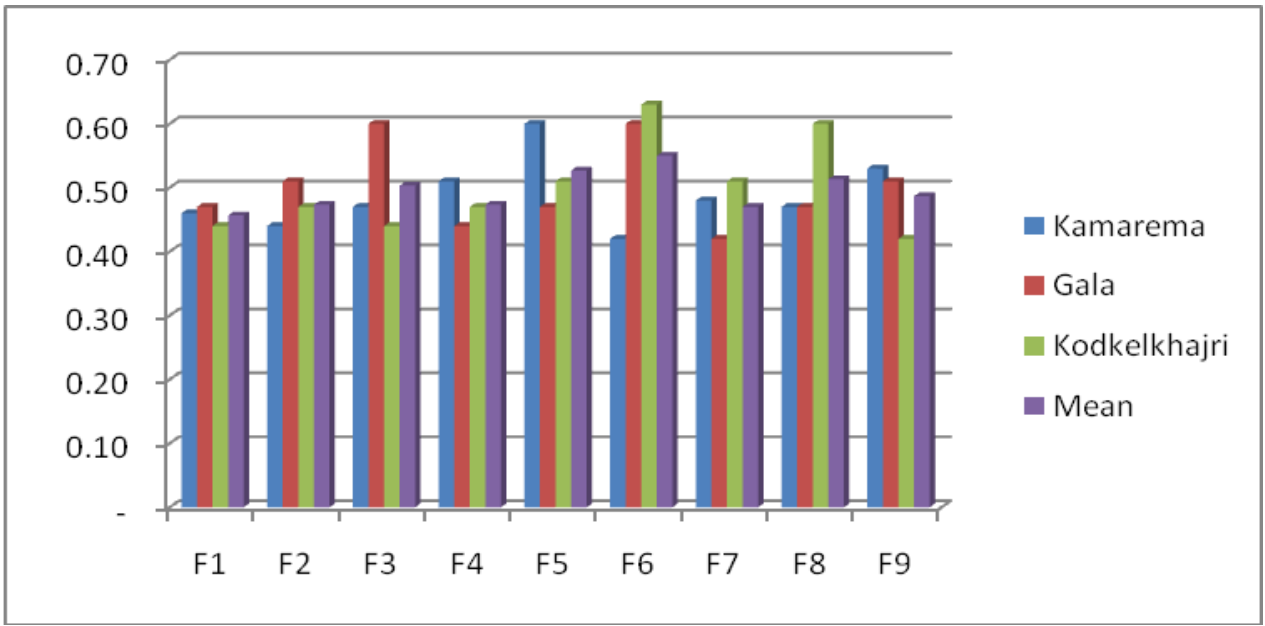

Fig.4 Availability of Nitrogen at Different Village of Jashpur District

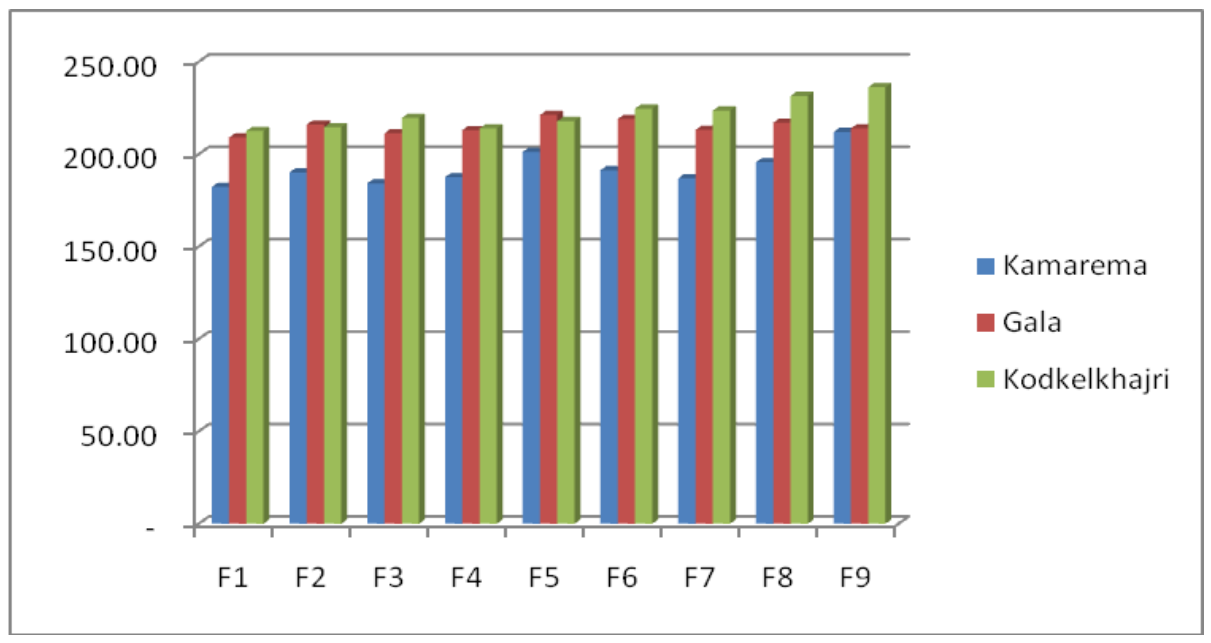


Fig.5 Availability of phosphorus at different village of Jashpur District

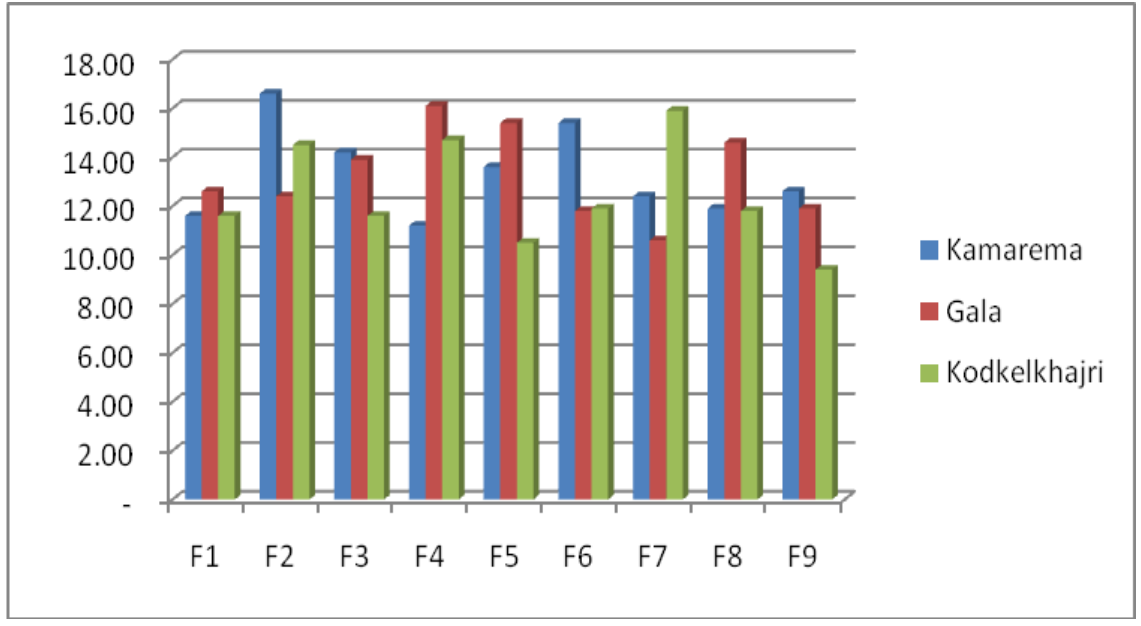

Fig.6 Availability of potassium at different village of Jashpur District

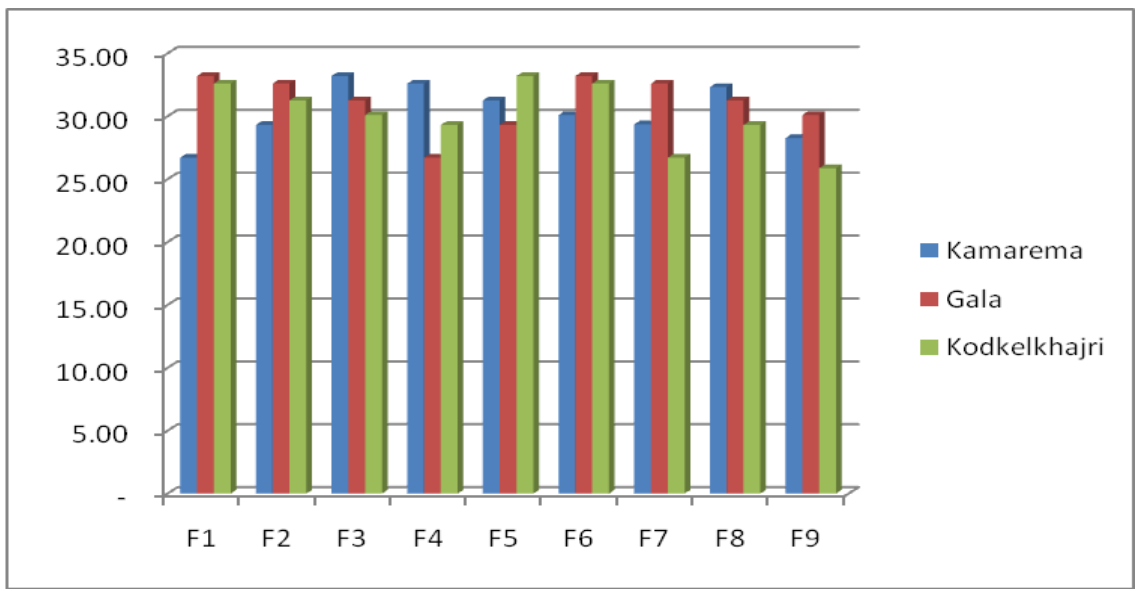

Fig.7 Availability of sulphur at different village of Jashpur District

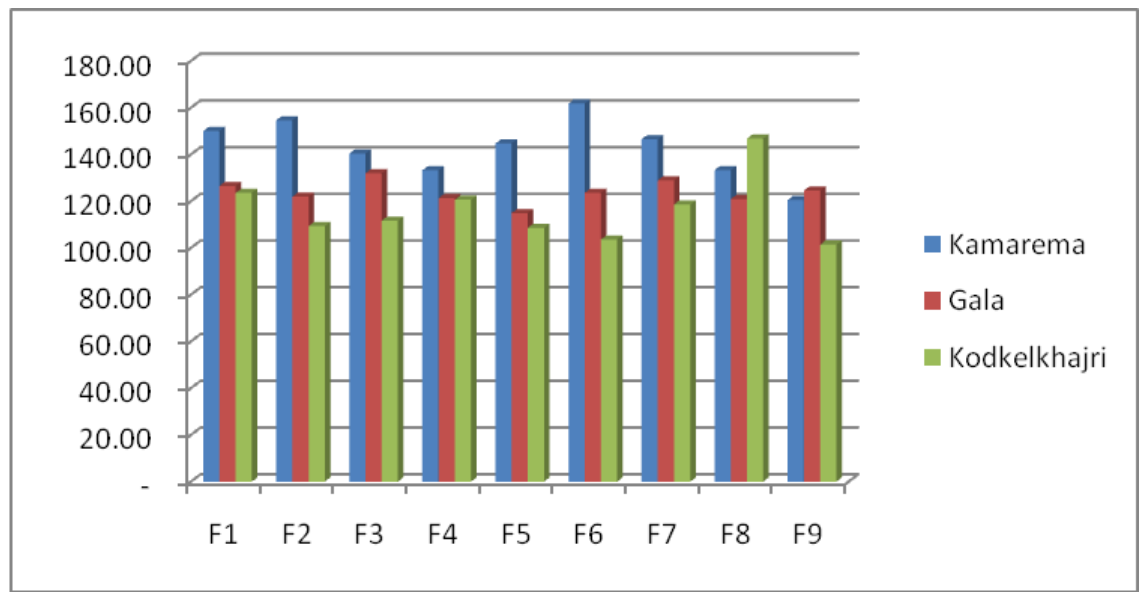


Fig.8 Availability of zinc at different village of Jashpur District

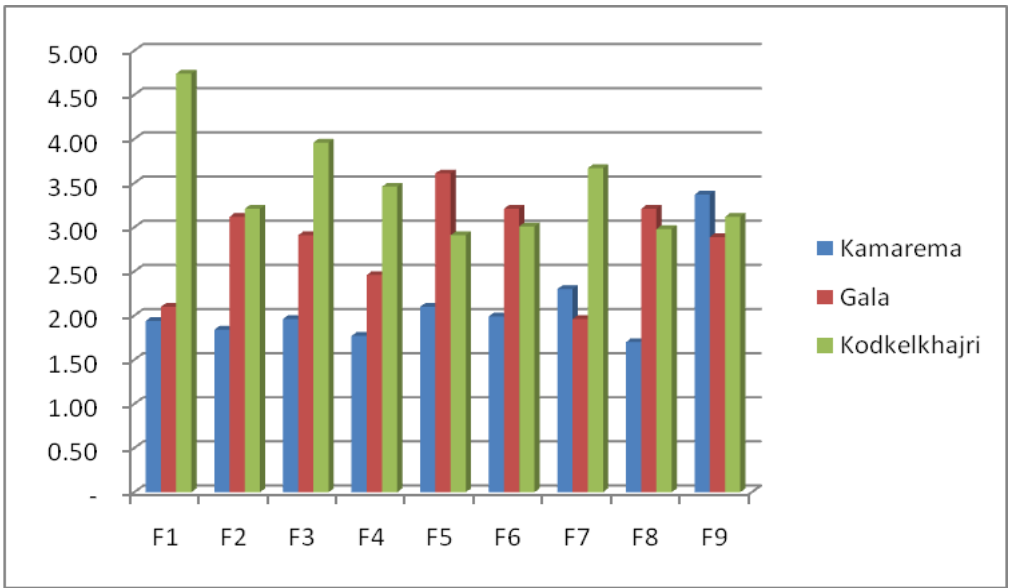

Fig.9 No. of siliqua on mustard crop at different village of Jashpur District

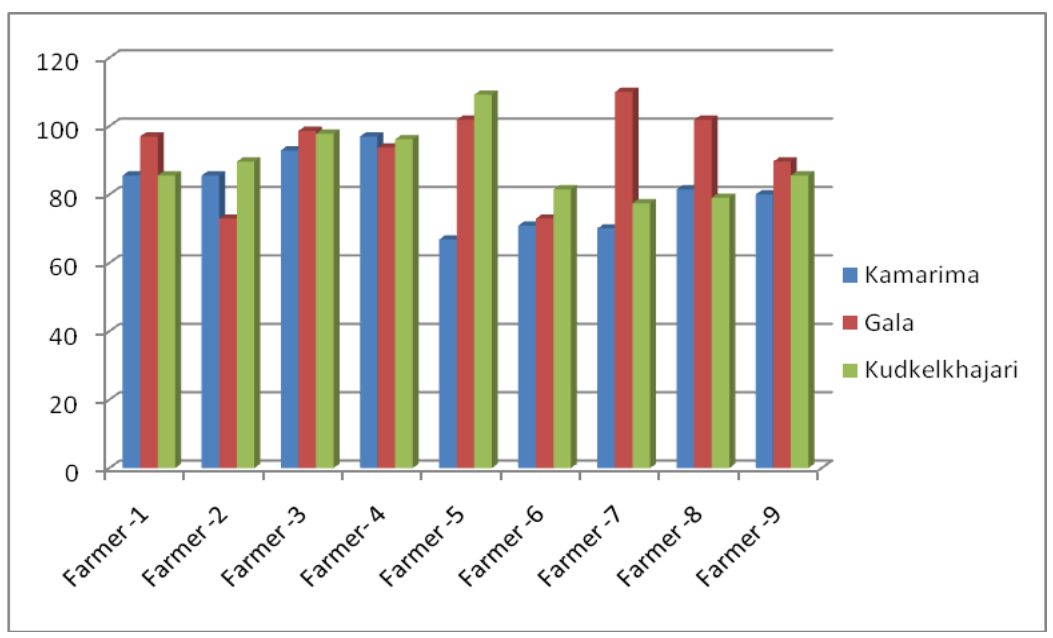

Fig.10 Mustard crop yield at different village of Jashpur District

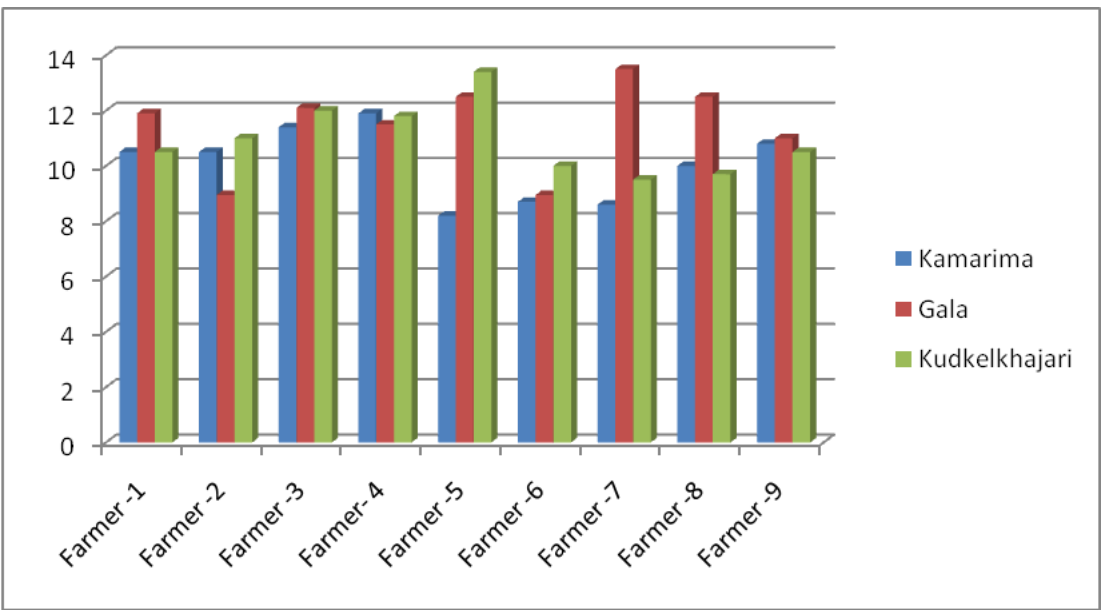


Available zinc $\left(\mathrm{kg} \mathrm{ha}^{-1}\right)$

The lowest value of zinc (ppm) is found in soil of Kamaremaat depth $(0-15 \quad \mathrm{~cm}) \quad 1.70$ $\mathrm{kg} \mathrm{ha}{ }^{-1}$ and highest value in soil of Kodkelkhajriat depth $(0-15 \mathrm{~cm}) 4.74 \mathrm{~kg} \mathrm{ha}^{-1}$.

The available zinc $\left(\mathrm{kg} \mathrm{ha}^{-1}\right)$ was found to be non-significant at different depths and villages (Fig. 8).

It can be concluded that the soil of Pathalgaon and Bagicha Block in Jashpur district of Chhattisgarh showed status according to Table 1. The value of $\mathrm{pH}$ is found varied from 5.42 to 6.51. It is neutral in nature, similarly results reported by (Meena et al., (2006). The EC value is varied from 0.04 to $0.16 \mathrm{dSm}^{-1}$ and it is normal, similarly results found (Upadhyay et al., 2014). The value of OC percent is found in varied from 0.44 to 0.63 per cent and this is low to medium level. The value of available nitrogen $\left(\mathrm{kg} \mathrm{ha}^{-1}\right)$ is found in varied from 182.2 to $236.25 \mathrm{~kg}$ ha- 1 is low to medium level similarly results reported by Pandey et al., (2013).

The value of available phosphorus $\left(\mathrm{kg} \mathrm{ha}^{-1}\right)$ is found in varied from 9.40 to $16.6 \mathrm{~kg} \mathrm{ha}^{-1}$ is medium level. The value of available potassium $\left(\mathrm{kg} \mathrm{ha}^{-1}\right)$ is found in varied from 101.4 to $170.3 \mathrm{~kg} \mathrm{ha}^{-1}$ is medium level similarly results reported by (Rao et al., 2012). The value of available zinc $\left(\mathrm{kg} \mathrm{ha}^{-1}\right)$ is found in varied from 1.70 to $4.74 \mathrm{~kg} \mathrm{ha}^{-1}$ is medium to high level. The value of available sulphur $\left(\mathrm{kg} \mathrm{ha}^{-1}\right)$ is found varied from 25.87 to $33.2 \mathrm{~kg}$ $\mathrm{ha}^{-1}$ is medium level (Table 2).

\section{Siliqua (per plant)}

The lowest value of Siliqua (per plant) is found in Mustard crop of Kamarema at 66.83 per plant and highest value in Mustard crop of Gala 110.02Siliquaper plant. The available Siliqua (per plant) was found to be significant at different villages (Fig. 9).

\section{Yield (Q/ha.)}

The lowest value of yield $(\mathrm{Q} / \mathrm{ha}$.) is found in Mustard crop of Kamaremaat 8.20 (Q/ha.) and highest value in Mustard crop of Gala 110.02 Yield (Q/ha.). The available yield (Q/ha.) was found to be significant at different villages (Table 3 and 4).

It is concluded that the best Cropping sequence with recommended doses of fertilizer also increased the fertility status of soil. The Maximum Mustard yield, Maximum Siliqua and best growth result of Mustard crop in Pathalgaon and Bagicha block of Jashpur district (Fig. 10).

\section{Abbreviations}

Q/ha.: Quintal/hactare

$\mathrm{Kg} / \mathrm{ha}$.: Kilogram/hactare

$\%$ : Percent

$\mathrm{dSm}^{1} .:$ Desi Simom Per Meter

$\mathrm{cm}$ : Centimeter

$\mathrm{mm}$ : Milimeter

PPM: Parts Per Million

$\mathrm{Ph}$ : Proton of Hydrogen

EC: Electrical Conductivity

OC: Organic Carbon

$\mathrm{N}$ : Nitrogen

P: Phosphorus

K: Potassium

Zn: Zinc

S: Sulphur

G: gram

Kcal: Kilo Calorie

\section{Acknowledgement/Funding resource}

We is thankful to Krishi Vigyan Kendra, Jashpur 496220, Indira Gandhi Krishi Vishwavidyalaya, Raipur and Support by all Famers to be conducted CFLD 


\section{References}

Dubey S. K., Tripathi S. K. and Singh, B. (2013) Effect of Sulphur and Zinc Levels on Growth, Yield and Quality of Mustard [Brassica juncea (L.) Czern\&Coss.]Research \& Reviews: A Journal of Crop Science and Technology, Volume (2), Issue 1, 2319-3395.

Goovaerts P. (1998) Geo-statistical tools for characterizing the spatial variability of microbiological and physic-chemical soil properties. Biol. Fertil. Soil, 27: 315-334.

Meena M.C., Patel K.P. and Rathod D.D. (2006) Effect of zinc iron and sulphur on mustard in loamy sand soil. Indian J. Fertilizer, 2 (5), 55-58.

Pandey G, G Misra, K Kumari, S Gupta, SK Parida, D Chattopadhyay and M Prasad (2013) Genomewide development and use of microsatellite markers for large-scale genotyping applications in foxtail millet [Setaria italica (L.)]. DNA Res. 20: 197-207.
Rao, S.R., Qayyum, A., Razzaq, A., Ahmad, M., Mahmood, I. \& Sher, A. (2012) Role of foliar application of salicylic acid and 1-tryptophan in drought tolerance of maize. Journal of Animal and Plant Sciences, 22, 768-772.

Tripathi and Bhardwaj (2016) Study the physico-chemical parameters in soil samples from Mungeli, Chhattisgarh, India. Asian J. Pharm. Res., 6(2), 8183.

Upadhyay Y., Swaroop N., Sahu Y. K., Dhruw S. S. and Verma P. D. (2016) Interaction effects of different doses of sulphur and zinc with NPK on physico -chemical properties of soil in yellow mustard (Brassica compestris L.) $\mathrm{Cv}$. Krishna Super Goldi. International Journal of Agricultural Science and Research (IJASR), vol. 6: 2250-0057.

Upadhyaya HD, SL Dwivedi, SK Singh, S Singh, M Vetriventhan and S Sharma (2014). Forming core collections in barnyard, kodo, and little millets using morphoagronomic descriptors. Crop Sci. 54: 2673-2682.

\section{How to cite this article:}

Alakh Gour, Dogendra Kumar Sahu and Deepak Thakur. 2019. Assessment of Soil for Increasing the Yield of Mustard in Northern Hills, Chhattisgarh. Int.J.Curr.Microbiol.App.Sci. 8(11): 2331-2341. doi: https://doi.org/10.20546/ijcmas.2019.811.270 